\title{
Title: A SKULL-BASED MULTIPLE DIPOLE PHANTOM FOR EEG AND MEG STUDIES
}

Author(s):

M. E. Spencer, R. M. Leahy, J. C. Mosher

Submitted to:

BIOMATNETISM '96, Santa Fe, NM, Feburary 1996

\section{Los Alamos} NATIONAL LABORATORY
PECEMPD

पU1 1929

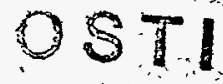

\section{DISTRBUTION OF THIS DOCUMENT IS URLMITED}
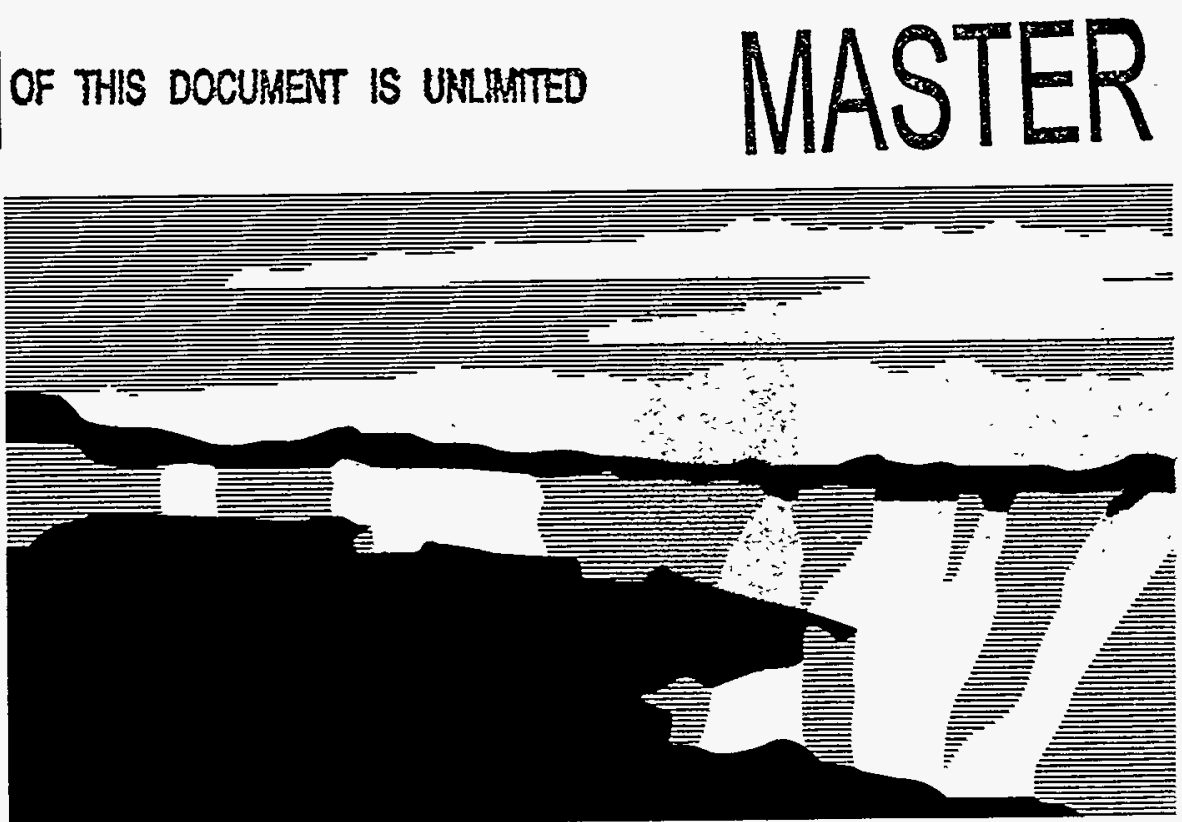

Los Alamos National Laboratory, an affirmative action/equal opportunity emplóyer, is operated by the University of California for the U.S. Department of Energy Los Alamos National Laboratory, an affirmative action/equal opportunity employer, is operated by the University of California tor the U.S. Departmententerive, royalty-free license to publish or reproduce the published form of this contribution, or to allow others to do so, for U.S. Government purposes. The Los Alamos National Laboratory requests that the publisher identify this article as work performed under the auspices of the U.S. Department of Energy. 


\section{DISCLAIMER}

This report was prepared as an account of work sponsored by an agency of the United States Government. Neither the United States Government nor any agency thereof, nor any of their employees, makes any warranty, express or implied, or assumes any legal liability or responsibility for the accuracy, completeness, or usefulness of any information, apparatus, product, or process disclosed, or represents that its use would not infringe privately owned rights. Reference herein to any specific commercial product, process, or service by trade name, trademark, manufacturer, or otherwise does not necessarily constitute or imply its endorsement, recommendation, or favoring by the United States Government or any agency thereof. The views and opinions of authors expressed herein do not necessarily state or reflect those of the United States Government or any agency thereof. 


\title{
A Skull-Based Multiple Dipole Phantom for EEG and MEG Studies
}

\author{
${ }^{1}$ Spencer, M.E., ${ }^{1}$ Leahy, R.M., and ${ }^{2}$ Mosher, J.C. \\ ${ }^{I}$ University of Southern California, Los Angeles, California, USA; ${ }^{2}$ Los Alamos \\ National Laboratory, Los Alamos, New Mexico, USA
}

\begin{abstract}
A versatile phantom for use in evaluating forward and inverse methods for MEG and EEG has been designed and is currently being constructed. The phantom consists of three major components: (i) a 32-element current dipole array, (ii) a PC-controlled dipole driver with 32 isolated channels allowing independent control of each dipole, (iii) spherical and human-skull mounts in which the dipole array is placed. Materials were selected throughout the phantom to produce minimal field distortions and artifacts to enable acquisition of high quality EEG and MEG data. The dipoles are made from a rigid narrow $(.84 \mathrm{~mm})$ stainless steel coax cable. The dipole drivers can be configured as either current or voltage sources, are independently programmable and fully isolated, and are capable of producing arbitrary bipolar waveforms up to a $200 \mathrm{~Hz}$ bandwidth. The spherical mount is a single shell sphere filled with conductive gelatin. The human skull mount has three shells: "brain" (conducting gelatin), "skull" (the skull is impregnated with a low conductivity conducting gelatin), and "scalp" (a thin layer of rubber latex mixed with $\mathrm{NaCl}$ to achieve a conductivity matched to the brain). The conductivities will be adjusted to achieve approximately an 80:1:80 ratio. Data collected to date from the spherical phantom shows excellent agreement between measured surface potentials and that predicted from theory (27 of the 32 dipoles give better than $99.9 \%$ rms fit) and negligible leakage between dipoles. We are currently completing construction of the skull mount.
\end{abstract}

\section{INTRODUCTION}

There has recently been great interest in developing accurate forward and inverse methods for mapping between neural current sources and the scalp potentials and external magnetic fields that they produce. Evaluation of these methods requires that data are available for which the "ground truth" is known. While the other functional modalities (fMRI and PET) offer the potential for providing ground truth for clinical and volunteer studies, these methods are currently not sufficiently well understood or mature to provide reliable validation. Evaluation can be performed using computer simulation. However, the majority of published results that use computer simulations assume simplified models for the head, instrumentation and noise. Typical simulations use a spherical head with point measurements of the scalp potential or magnetic field and additive white Gaussian noise. To establish realistic limits on the performance of forward and inverse methods, more realistic models are required. While it is possible to develop more elaborate simulations, there are advantages to performing evaluation using data collected directly from a physical system. Such studies have been performed using dipolar sources implanted in epilepsy patients [1], however the procedures required to implant these sources (e.g. temporary removal of part of the skull, and related tissue displacement and swelling) may result in severe distortion of volume currents. While such studies are important, they are not ideal for evaluation of general forward and inverse methods. Interesting studies have been performed with dipoles implanted in a cadaver head [2] and a gelatin filled skull [3], but these studies have been restricted to at most a few dipoles. A multiple dipole phantom was used in [4] for evaluation of several MEG imaging methods. This is a dry phantom that is based on the theoretical description in [5] in which it is shown that the resulting fields are identical to those produced by a current dipole in a uniformly conducting medium. The major limitation of this phantom is that is unable to generate the volume currents associated with realistic head geometries nor is it suitable for EEG.

Motivated by the desire to produce realistic data corresponding to complex spatio-temporal current sources and to include the effects of realistic head geometries, we have designed and fabricated a multiple dipole phantom consisting of 32 independently programmable and isolated dipoles which can be inserted in either a spherical or skull mount and used to collect both EEG and MEG data. In the following section we describe the phantom. We then report on the current status of the project and briefly summarize our preliminary results.

This work was supported by the National Institute of Mental Health, Grant No. R01-MH53213. 

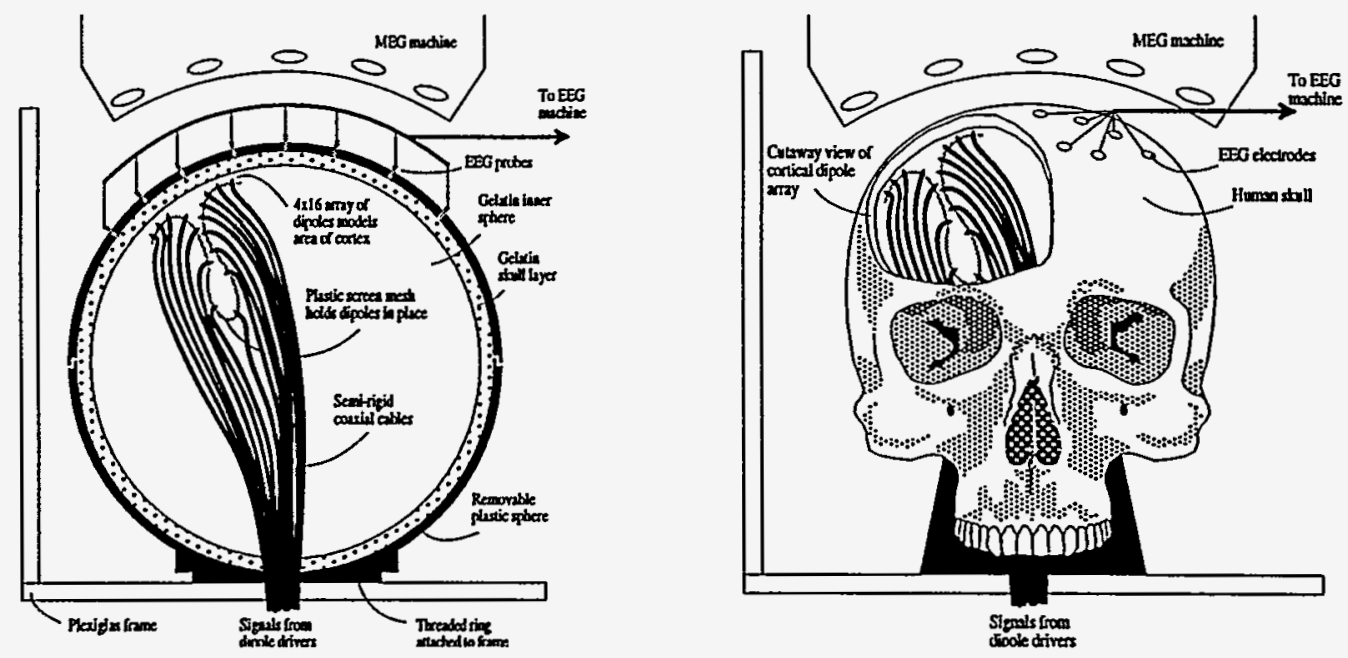

Fig. 1. Schematics for the spherical and skull phantoms.

\section{PHANTOM DESIGN}

The phantom design consists of three components: (i) a 32-element current dipole array, (ii) a PC-controlled dipole driver with 32 isolated channels allowing independent control of each dipole, (iii) spherical and human-skull mounts in which the dipole array is placed. We describe each of these three components below.

\section{Dipole Array}

The dipoles were constructed from semi-rigid coaxial cable (UT-034-SS-SS, Micro-Coax, UTI, Collegeville, PA) consisting of a $0.84 \mathrm{~mm}$ outside diameter shield made from stainless steel alloy (\#304) tubing, a Teflon PTFE (Polytetrafluoroethylene) insulator and an inner stainless steel conductor. The semi-rigid coax was insulated using

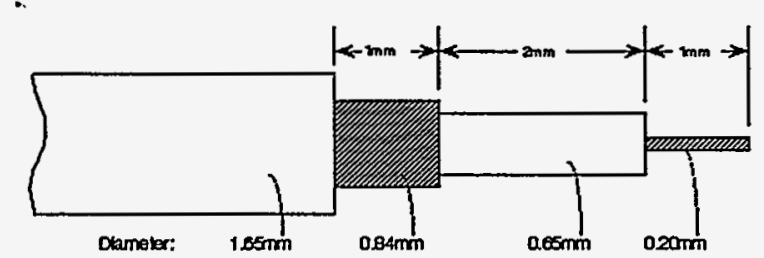
standard 3/64 inch heat shrinkable tubing and then striped back to expose $1 \mathrm{~mm}$ of the shield and $1 \mathrm{~mm}$ of the inner conductor to the volume conducting medium, Fig. 2. Thirty two dipoles were constructed and arranged to approximately follow a pair of sulcal folds: 16 were distributed along, and arranged normally to, a nominal central sulcus. Another 14 dipoles were arranged along a Fig. 2. Dimensions of the stainless steel coaxial dipole. nominal calcerine fissure. The remaining dipoles were positioned to represent a pair of frontal sources. The semi-rigid nature of the cable allows us to bend the cable to individually position the dipoles for the desired locations and orientations. Once the dipoles are positioned, we can move the dipole array from the spherical to the skull mount with no significant change in the relative dipole locations.

\section{Driver Design}

The dipoles are driven by isolated current sources which in turn are controlled through a special purpose interface to a personal computer. Arbitrary waveforms can be generated simultaneously at each of the 32 dipoles. These waveforms are generated using standard MATLAB code on the $\mathrm{PC}$ and directed to the dipole drivers. The disk file on our PC can contain over an hour of real time data with a sampling rate of 1000 samples per second per channel. Streamer software and the PDMA-16 Digital DMA Board (Keithley Metrabyte, Taunton, MA 02780) in the PC is used to clock the data at a constant rate in 16 bit words to the external dipole driver chassis.The electronics for the 32 isolated dipole drivers is housed in a 19 inch rack mount chassis and powered by the PC's 5V power supply. A six foot 37 conductor ribbon cable carries the 16 bit data words and clock signals from the PDMA-16 board in the PC to 
the dipole drivers, Fig. 3. A parallel interface and clock generator format the digital data for 32 electrically isolated Digital-to-Analog (D/A) converters. The sampling rate clock is divided by 1000 and is supplied to the EEG or MEG machine for synchronization when performing epoch-based acquisition.

The 32 channels are electrically isolated from ground and each other. Each channel has optical isolators for the serial data, clock and load signals and has a transformer isolated DC-to-DC power converter, Fig. 3. The digital data is converted to an analog signal using a 12bit D/A converter (Linear Technology, LTC1257). The signal from the $\mathrm{D} / \mathrm{A}$ converter is then filtered with a second order bandpass filter with a passband of $1 \mathrm{~Hz}$ to $200 \mathrm{~Hz}$ and a transconductance amplifier is used to convert the voltage signal to a current in the dipole. The dipole is driven with a current source so that the resulting dipole current is independent of the impedance of the dipole. Alternatively, voltage output can be selected with a jumper change.

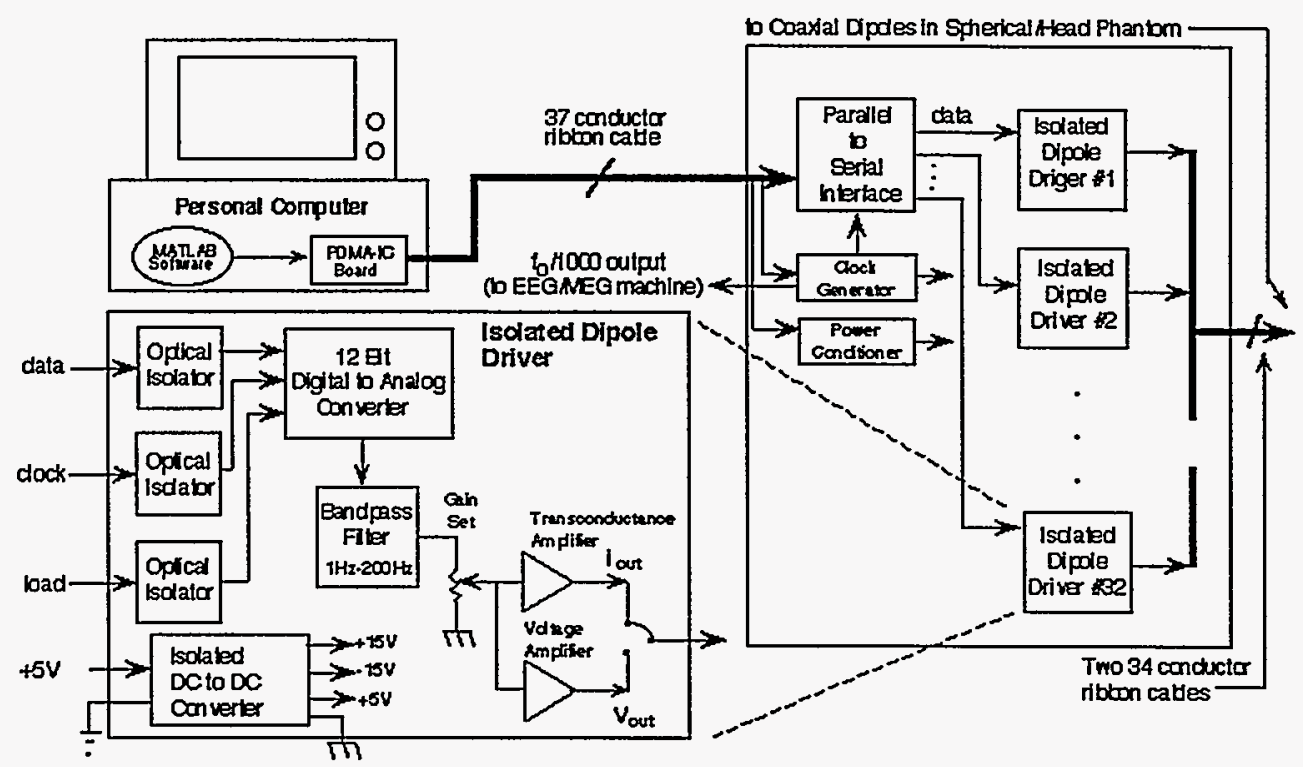

Fig. 3. The dipole driver chassis receives real-time digital signals from the $\mathrm{PC}$ and is formatted to drive 32 electrically isolated dipole driver circuits.

\section{The Spherical and Skull Mounts}

The single layer spherical phantom is constructed within a plastic sphere comprising two hemispheres that snap together to form a tight seal. The spherical single shell phantom will be used to validate the dipolar patterns of the coaxial sources and electrical isolation when running multiple dipoles. We can also compare results for identical source patterns for the spherical and skull mounts to study the effect of the non-spherical geometry on the accuracy of our inverse solutions. The plastic sphere (Mangelsen's, Omaha, NE) has an inside diameter of $136.0 \mathrm{~mm}+0.2 \mathrm{~mm}$ and a wall thickness of $1.9 \mathrm{~mm}+-0.4 \mathrm{~mm}$. The lower half is mounted to the base with a removable slip ring and the upper half is fitted with 34 silver silver-chloride electrodes (Neuromedical Supplies, Herndon, Va) including reference and a ground electrode. The electrodes are located in an irregular pattern over the sphere. The reference probe is positioned at the top dead center. Prior to data collection, the sphere was filled with a conductive gelatin made from water, sodium chloride (to control conductivity), Knox unflavored Gelatin (to solidify the solution), and dimethyl benzyl ammonium chloride (to prevent bacteria growth).

To model the irregular, eccentric morphology of the head, a human skull mount is currently under construction. The whole skull will be boiled in saline solution and gelatin to impregnate the bone to achieve an appropriate conductivity. The conductivity will be adjusted by changing concentrations of sodium chloride. Conductivity will be measured using samples of the prepared solution. Conductivity of the skull can be determined using calipers and resistance measurements at several places. The skull will then be filled with saline-gelatin mixture to represent the brain volume; the conductivity will be adjusted to provide an approximate 80:1 brain/skull ratio. To provide a scalp layer, the outside of the skull will be coated with rubber latex molding compound (GP 306, Gerisch Products, Inc. 
20814 S. Normandy Ave. Torrance, CA90502). The latex will be mixed with sodium chloride to achieve the desired conductivity. X-ray CT images of the completed skull phantom will be collected to verify the dipole locations and to extract the skull and scalp surfaces.

The spherical and skull phantoms can be interchangeably attached to a plexi-glass base containing the dipole array. The base has a fill/drain plug for filling the brain volume of the phantom. A pressurized air volume in the neck pushes on a membrane that is in contact with the conducting solution to compensate for volume loss due to the small evaporation through the skull. Without volume compensation, evaporative losses through the skull and scalp layers can cause air gaps to form that will interrupt the volume currents in the skull. All materials in the base and phantoms are nonferrous. In Fig. 4 we show the various components of the phantom. We have collected 32 channel EEG data from the spherical phantom using a Neuroscan 32 channel EEG Synamps system. Of the 32 dipoles, 27 give better than a $99.9 \% \mathrm{rms}$ fit between the measured and theoretical data based on a least squares single dipole fit to each coaxial dipole. The fit for the remaining 5 dipoles range from $86 \%$ to $94 \%$. We believe these errors are primarily due to disruption of the volume currents by adjacent coaxial wires and can be reduced by repositioning the dipoles. We will then fill the skull mount and collect MEG and EEG data on the completed skull phantom.

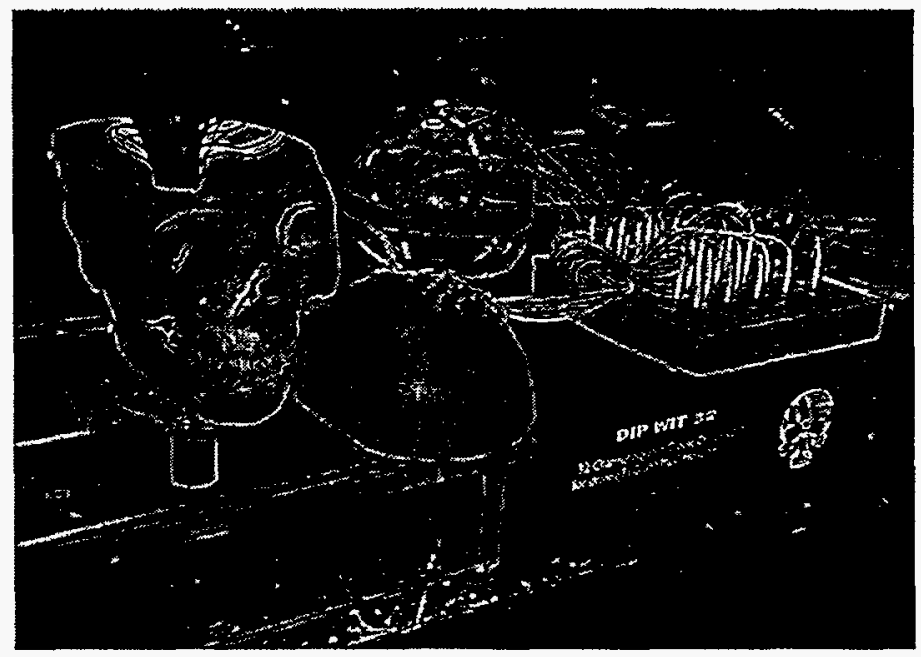

Fig. 4. Photograph showing from left to right: skull mount on plexi-glass base with dipole array inside the skull; skull cap covered with latex scalp layer and EEG electrodes attached; spherical mount with $\mathrm{Ag} / \mathrm{AgCl}$ electrodes attached; and the dipole driver chassis (DIPWIT32) below the head box for a 32 channel Synamps EEG system.

The authors would like to thank Jeff Lewine of the New Mexico Institute of Neuroimaging in Albuquerque for his valuable advice on the design of the skull phantom.

\section{REFERENCES}

[1] Cohen D, Cuffin BN, Yunokuchi K, Maniewski R, Purcell C. Cosgrove GR, Ives J, Kennedy JG, Schomer DL. 1990, "MEG versus EEG localization test using implanted sources in the human brain," Ann Neurol, 28:811817.

[2] Barth D, Sutherling W., Broffman J., Beatty, J., "Magnetic localization of a dipolar current source implanted in a sphere and a human cranium," Electroenceph Clin Neurophysiol., vol 63, pp 260-273, 1986.

[3] Lewine, JD, Edgar, JC, Repa, K, Paulson, K, Asture, RS, Orrison, WW Jr, "A Physical Phantom for Simulating the Impact of Pathology on Magnetic Source Imaging," Biomagnetism: Fundamental Research and Clinical Applications, eds C. Baumgartner et al. Elsevier,1995, pp. 368 - 372.

[4] Leahy, R.M., Mosher, J.C., and Phillips, J.W, "A comparative study of minimum norm inverse methods for MEGimaging," (this volume)

[5] Ilmoniemi, R.J., Hamalainen, M.S., Knuutila, J, 1985, "The forward and inverse problems in the spherical model," in Biomagnetism: Applications and Theory, eds Weinberg, Stroink, and Katila, Pergamon: New York, pp.278-282. 